\title{
Correction to: Attendance to cervical cancer screening among Roma and non-Roma women living in North-Western region of Romania
}

\author{
Trude Andreassen ${ }^{1,2,5}$ (1) $\cdot$ Adriana Melnic ${ }^{3} \cdot$ Rejane Figueiredo $^{4} \cdot$ Kåre Moen $^{5} \cdot$ Ofelia Şuteu $^{6,7} \cdot$ \\ Florian Nicula ${ }^{7} \cdot$ Giske Ursin $^{1,2,8} \cdot$ Elisabete Weiderpass ${ }^{1,4,9,10}$
}

Published online: 14 June 2019

(C) Swiss School of Public Health (SSPH+) 2019

\section{Correction to: International Journal of Public Health https://doi.org/10.1007/s00038-018-1107-5}

The authors would like to correct the errors in the publication of the original article. The corrected details are given below

(a) Page 617: Figure title: number 464 should be corrected to 463 .
The original article can be found online at https:// doi.org/10.1007/s00038-018-1107-5.

Trude Andreassen

trude.andreassen@kreftregisteret.no

1 Cancer Registry of Norway, Institute of Population-Based Cancer Research, Majorstuen, Post-Box 5213, 0304 Oslo, Norway

2 Institute of Basic Medical Sciences, University of Oslo, Oslo, Norway

3 The Romanian Cancer Society, Cluj-Napoca, Romania

4 Folkhälsan Research Center, Genetic Epidemiology Group, Helsinki, Finland

5 Institute of Health and Society, University of Oslo, Oslo, Norway
Corrected Figure:

Fig. 1 Barriers to screening participation among $46 \underline{3}$ never attending Roma and non-Roma women living in the North-Western region of Romania in 2016-2017.
6 University of Medicine and Pharmacy, "Iuliu Haţieganu", Cluj-Napoca, Romania

7 Institute of Oncology "Prof. Dr. Ion Chiricuţă" Cluj-Napoca (IOCN), Cluj-Napoca, Romania

8 Department of Preventive Medicine, University of Southern California, Los Angeles, CA, USA

9 Department of Community Medicine, Faculty of Health Sciences, University of Troms $\varnothing$, The Arctic University of Norway, Troms $\emptyset$, Norway

10 Department of Medical Epidemiology and Biostatistics, Karolinska Institutet, Stockholm, Sweden 


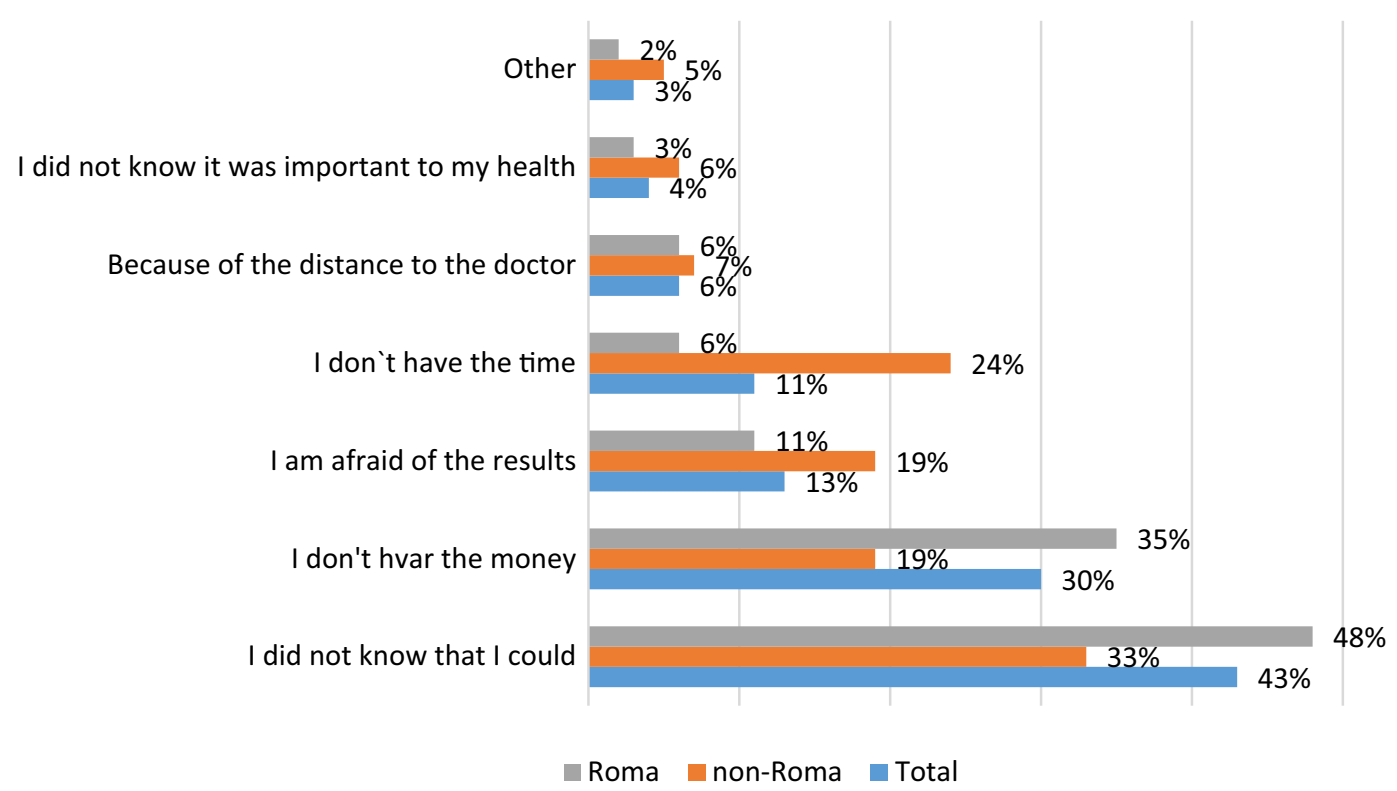

(b) Page 612: "The most frequent barrier for non-attendance among never-attenders was lack of awareness about the programme's existence (43\%)"should be changed to: "The most frequent barrier for non-attendance among never-attenders was that women did not know that they could take a screening test $(43 \%)$ ".

(c) Page 612: "lack of money (31\%)" should be corrected to "lack of money $(30 \%)$ ".

(d) Page 612: "and the distance to the doctor (11\%)" to "and the distance to the doctor (6\%)".

(e) "Roma women reported the three most frequent barriers (lacking awareness about the programme, lack of money and being afraid of results) more often than non-Roma women did. Non-Roma women, on the other hand, reported the barriers, time contains and the distance to the doctor, more frequent than Roma women did (Fig 1)."

These two sentences should be changed to:

"Roma women reported the two most frequent barriers (not knowing that they could take a screening test and lack of money) more often than non-
Roma women did. Non-Roma women, on the other hand, reported the barriers, time contains and being afraid of results, more frequent than Roma women did (Fig 1)."

(f) Page 616: "As much as $31 \%$ of women" to "As much as $30 \%$ of the women".

(g) Page 618: The most important barriers to screening participation were lack of knowledge about the programme's existence and lack of money (Fig. 1).—-should be changed to: "The most important barriers to screening participation were that women did not know that they could take a screening test and lack of money (Fig. 1).

(h) Page 615, Table 3: The Confidence Interval "4.14-2.53". This should be "1.14-2.53".

(i) Page 612: “(1.70, 4.14-2.53)" to "(1.70, 1.14-2.53)".

Publisher's Note Springer Nature remains neutral with regard to jurisdictional claims in published maps and institutional affiliations. 